A VIBEYOR

\title{
First Principles Study of Thermo-Mechanical Properties of Gallium Phosphide
}

\author{
Dotun Oladimeji' , Linu Malakkal', Barbara Szpunar', Ericmoore Jossou² and Jerzy \\ Szpunar ${ }^{2}$
}

${ }^{1}$ Department of Physics and Engineering Physics, University of Saskatchewan, Saskatoon, Canada

${ }^{2}$ Department of Mechanical Engineering, University of Saskatchewan, Saskatoon, Canada

\begin{abstract}
Using first principles approach, we calculated the structural, elastic, thermal and thermodynamic properties of semiconducting Gallium Phosphide (GaP). Quantum Espresso (QE) code with planewave pseudopotential approach to Density Functional Theory (DFT) and the more recently developed Generalized Gradient Approximation (GGA) scheme for solids (PBE sol) was utilized. The calculated lattice constant, elastic constants, bulk modulus, and Young's modulus are in excellent agreement with the known experimental values. The electron density of states revealed the hybridization between $\mathrm{Ga}(2 \mathrm{p})$ and $\mathrm{P}(2 \mathrm{p})$ and their covalent bonding. We also calculated the phonon dispersion relationship of GaP using the Density Functional Perturbation Theory (DFPT) as implemented in QE and the obtained phonon dispersion compares well with the available experimental results. Further, we calculated the lattice-assisted properties such as volume thermal expansion and heat capacity within Quasi-Harmonic Approximation (QHA) and the predicted properties are consistent with the experimental data. Finally, we presented the phonon contribution of thermal conductivity as a function of temperature up to $1400 \mathrm{~K}$ using the Slack model and Boltzmann Transport Equation (BTE).
\end{abstract}

\section{Keywords}

GaP, First principles, Electronic structure, Thermal conductivity, BTE, Slack model

\section{Introduction}

$\mathrm{GaP}$ is a semiconducting material that finds application in high power electronic devices such as Light Emitting Diodes (LEDs). With the advancement in semiconducting technologies, and the growing need for smaller and faster devices the manipulation of the material properties has become indispensable. For smaller and faster devices the unrestrained increase in temperature is apparently a constricting strand for the device behavior and dependability. Hence, an efficient heat withdrawal is essential for the performance and reliability of GaPbased electronic devices [1].
Moreover, the thermophysical properties are fundamental characteristics to be considered in selecting the cooling schedule of a GaP-based device. Therefore to improve the reliability and efficiency of $\mathrm{GaP}$ devices, it is important to evaluate the thermal properties of $\mathrm{GaP}$ up to relatively high temperatures. Some previous works had determined properties such as indirect band gap [2], thermal expansion [3] and thermal conductivity [4] at lower temperatures. Theoretically, some previous works have used first principles approach to determine thermal transport properties of $\mathrm{GaP}$ and other semiconducting materials. Nina Shulumba, et al. [5] determined the effect of harmonicity on the thermal conductivity of Al-

*Corresponding author: Dotun Oladimeji, Department of Physics and Engineering Physics, University of Saskatchewan, Saskatoon, S7N 5E2, Canada, E-mail: dotun.john@usask.ca

Received: April 12, 2017: Accepted: August 21, 2017: Published: August 24, 2017

Copyright: (C) 2017 Oladimeji D, et al. This is an open-access article distributed under the terms of the Creative Commons Attribution License, which permits unrestricted use, distribution, and reproduction in any medium, provided the original author and source are credited. 
Table 1: Structural and mechanical properties of cubic GaP at $0 \mathrm{~K}$ and $300 \mathrm{~K}$ evaluated by QHA in comparison with other DFT calculations $[6,25]$ and experiments $[18,19]$. The lattice parameter $\left(a_{o}\right)$ is in $\AA$, the mechanical properties (elastic constants $\left(C_{11}\right.$ $C_{12}$ and $C_{44}$ ), Bulk modulus $(B)$, Shear modulus $(G)$ and Young modulus $(Y)$ ) are in GPa, and the Poisson ratio $(\eta)$ has no unit.

\begin{tabular}{|l|l|l|l|l|l|l|}
\hline Properties & PBESOL (0 K) & PBESOL (300 K) & Ref [6] (0 K) & Ref [25] (0 K) & Exp. [18] & Exp. [19] \\
\hline Lattice parameter $(\AA)$ & 5.449 & 5.454 & 5.502 & - & 5.451 & 5.451 \\
\hline $\mathrm{C}_{11}(\mathrm{GPa})$ & 130.3 & 129.2 & 123 & 137.5 & - & - \\
\hline $\mathrm{C}_{12}(\mathrm{GPa})$ & 56.6 & 55.9 & 50.9 & 59.4 & - & - \\
\hline $\mathrm{C}_{44}(\mathrm{GPa})$ & 88.9 & 87.8 & 70.9 & 72.2 & - & - \\
\hline Bulk modulus $(\mathrm{B})(\mathrm{GPa})$ & 81.2 & 80.3 & 75.1 & - & 88.7 & - \\
\hline Shear modulus (G) $(\mathrm{GPa})$ & 68.1 & 67.4 & 54.2 & - & - & - \\
\hline Young modulus (Y) (GPa) & 149.2 & 147.7 & - & - & - \\
\hline Poisson ratio $(\mathrm{\eta})$ & 0.1939 & 0.1937 & - & - & - \\
\hline
\end{tabular}

uminium Nitride $(\mathrm{AlN})$ at relatively high temperature using the GGA Perdew-Burke-Ernzerhof (PBE) functional. Liu li, et al. [6] have investigated the phase transition, elastic and thermodynamic properties of GaP by the DFT using the Perdew-Wang (PW91) version of the GGA exchange-correlation functional. Rashid Ahmed, et al. [7] studied the structural and electronic properties of group III phosphides, including GaP, using the full potential linearized augmented plane wave method within the Local Density Approximation (LDA). Previously, Lindsay, et al. [8] used the BTE to predict the Lattice Thermal Conductivity $\left(\mathrm{k}_{\mathrm{L}}\right)$ of $\mathrm{GaP}$ and other semiconductors at low temperature $(<400 \mathrm{~K})$ using the LDA norm-conserving pseudo potentials. However, the data on transport properties of $\mathrm{GaP}$ at high temperature are limited. Therefore, in this work, we used the first principles calculation to evaluate the thermal properties of $\mathrm{GaP}$ at relatively high temperature.

\section{Methodology}

We performed the first principles calculation by using Quantum ESPRESSO (QE) [9] and also utilized an interface QE_NIPY-advanced developed by our group [10] to run the code andanalyze outputs. To determine the equilibrium structural properties, we performed structural optimization by varying cell parameters and atomic positions such that the difference of total Energy (E) converged to less than one meV. To evaluate the Bulk modulus $\left(\mathrm{B}_{\mathrm{o}}\right)$, we fitted the total Energy (E) versus Volume $(\mathrm{V})$ using the third order Birch-Murnaghan equation of state [11]. We calculated the Phonon Density of States (PDOS) that includes all the phonons over the entire Brillouin zone needed to estimate the phonon-assisted properties by using Density Functional Perturbation Theory (DFPT). All the lattice assisted properties such as fixed volume Heat Capacity $\left(\mathrm{C}_{\mathrm{v}}\right)$, and Volume Thermal Expansion $\left(\alpha_{\mathrm{v}}\right)$ were calculated within QHA [12]. The phonon contribution to $\mathrm{k}_{\mathrm{L}}$ is predicted using the Slack model [13]. The detailed description of the methodology and the equations used to calculate all the properties mentioned above can be found in our previous work $[14,15]$ and is not repeated here for brevity. Moreover, we have found in our previous work [15] that the new PBE sol potential for solids predict material properties in better agreement with the experiments and therefore we have used the same potential for this work. We also compare the $\mathrm{k}_{\mathrm{L}}$ by Slack method with the solutions of BTE. To solve BTE we used the recently developed software package (Sheng BTE) by Wu Li, et al. [16].

\section{Computational Details}

All our calculation uses the norm-conserving PBE sol (new exchange-correlation functional for solids) [17] functional. We obtained the total energy convergence using a Monkhorst-Pack sampling of $10 \times 10 \times 10$ and 100 Ry plane wave's cut-off energy. On a mesh of $5 \times 5 \times 5$ q-points, we calculated the dynamical matrices for phonon density of states, in the irreducible Brillouin zone.

We considered a super cell of $4 \times 4 \times 4$ to calculate the third order Inter atomic Force Constants (IFCs) using sheng BTE. The calculated third order IFCs was used for solving BTE. The force cutoff distance was set such that the interaction range is up to the three nearest neighbors. A mesh of $5 \times 5 \times 5$ q-points was used to calculate the second order IFCs by QE and needed by BTE to compute the $\mathrm{k}_{\mathrm{L}}$ of $\mathrm{GaP}$.

\section{Results and Discussions}

\section{Structural and mechanical properties}

Listed in Table 1 are the calculated ground state structural and mechanical properties of cubic GaP in comparison with experimental values obtained from refs. $[18,19]$ at room temperature. Table 1 also provides the calculated structural and mechanical properties of $\mathrm{GaP}$ at $300 \mathrm{~K}$ within QHA. At room temperature $(300 \mathrm{~K})$, the calculated lattice constant shows excellent agreement with experiments $[18,19]$. The cubic structure GaP has three independent elastic constants: $\mathrm{C}_{11}, \mathrm{C}_{12}$, and $\mathrm{C}_{44}$ and to obtain the single crystal stiffness constants we used the stress-strain method [20,21] implemented in the QE_nipy-advanced interface [10]. From the calculated stiffness constants, using the Voigt-Reuss-Hill averaging scheme [22-24], we determined the Bulk Modulus (B), 
Shear Modulus (G), Young's Modulus (Y), and the Poisson's Ratio $(\eta)$. All the predicted mechanical properties (as shown in Table 1) agree well with the experimental values. We also note that the new PBE sol functional predicted the structural and mechanical properties more accurately than the previous DFT calculations $[6,25]$ using the PW GGA exchange-correlation function. For example, the bulk modulus we predicted, using Voigt-Reuss-Hill averaging scheme, which is $80.3 \mathrm{GPa}$ is more accurate than the previous prediction of $75 \mathrm{GPa}$ when compared to the experimental value of $88.7 \mathrm{GPa}$ [18]. In this work, we used the revised Perdew-Burke-Ernzerh of (PBEsol) Generalized Gradient Approximation (GGA) functional because it improves the equilibrium properties of densely packed solids and their surfaces. More detailed descriptions regarding the underlying physics of the PBE sol functional is provided in ref. [26]. Since the agreement of calculated and experimental properties is essential, we, therefore, study the electronic density of

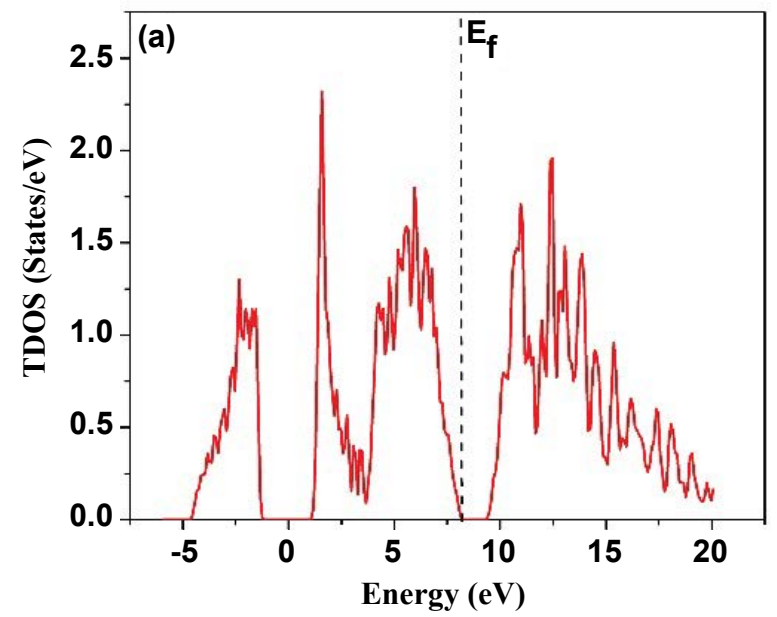

state, phonon dispersion, and thermodynamic properties of $\mathrm{GaP}$ using the same PBE sol functional.

\section{Electronic structure}

Figure 1a and Figure 1b shows the Total Density of States (TDOS) and Partial Density of States (PDOS) of $\mathrm{GaP}$ respectively. Figure 1a clearly indicates that $\mathrm{GaP}$ is a semiconductor. Although, the calculated band gap (1.7 $\mathrm{eV})$ is better than the previous DFT calculation $(1.44 \mathrm{eV})$ using Local Density Approximation (LDA) potential [7], but when compared to the experimental value of 2.35 $\mathrm{eV}$ [27] our prediction is under estimated. The PDOS (shown in Figure $1 \mathrm{~b}$ ) indicates a hybridization of $\mathrm{Ga}(2 \mathrm{p})$ and $\mathrm{P}(2 \mathrm{p})$ which accounts for the covalent bonding of $\mathrm{GaP}$. The Fermi energy of $\mathrm{GaP}$ is evaluated to be $8.25 \mathrm{eV}$.

\section{Phonon dispersion and thermodynamic properties}

The phonon vibrational spectra are the fingerprint of materials. The frequency of vibration is the measure

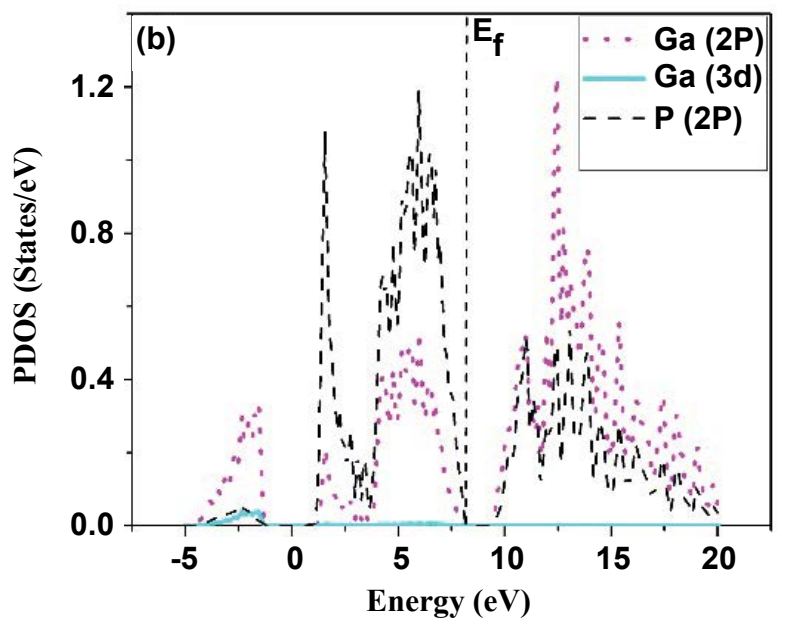

Figure 1: a) TDOS for GaP; b) PDOS of Ga and P atoms.
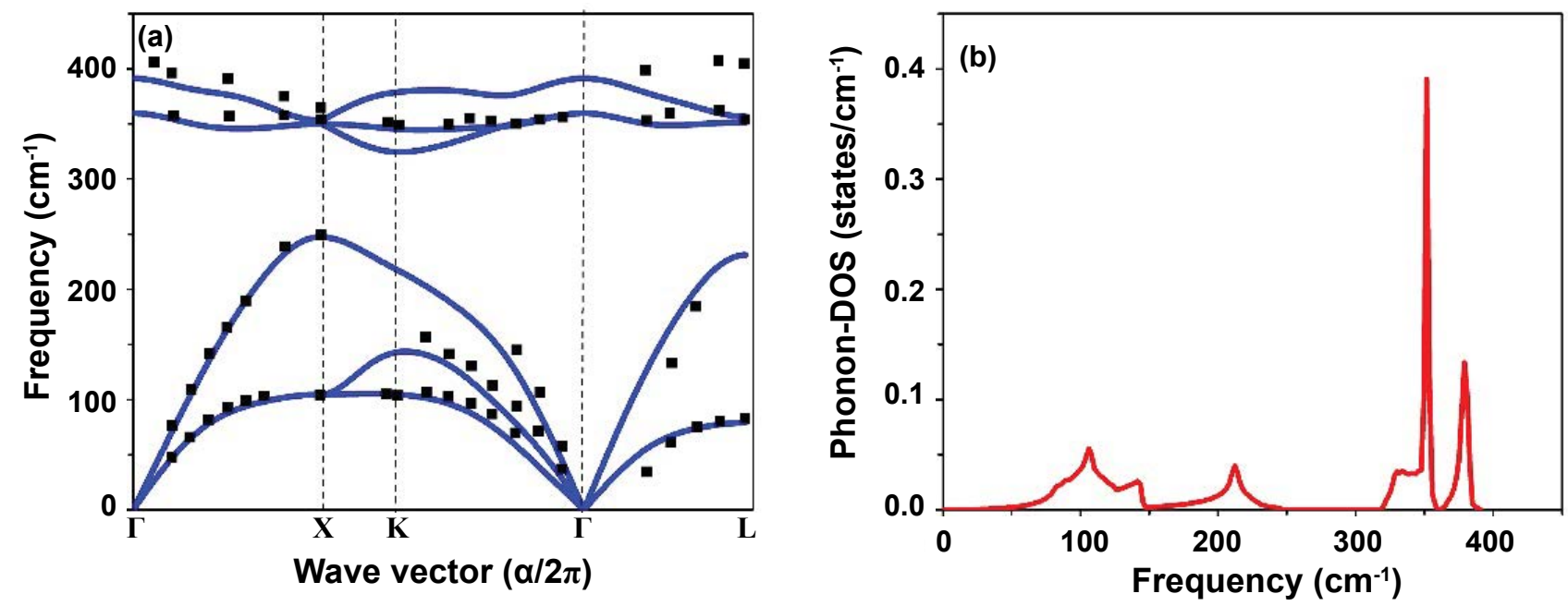

Figure 2: a) Calculated phonon dispersion curve of the gallium phosphide cubic structure at $300 \mathrm{~K}$. The solid lines show the theoretical prediction and the black squares are the experimental points measured by Borcherds, et al. [28] at $300 \mathrm{~K} ; \mathrm{b}$ ) Shows the phonon density of states for GaP. 
of the stiffness of a bond and also a function of atomic mass. Therefore, knowledge of phonon dispersion in the entire Brillouin zone is necessary to understand the thermodynamic properties, thermal expansion, and thermal conductivity. In Figure $2 \mathrm{a}$ we present the phonon dispersion of $\mathrm{GaP}$ at its equilibrium volume along the high symmetric $\Gamma-\mathrm{X}-\mathrm{K}-\Gamma-\mathrm{L}$ directions. GaP has two (n) atoms in its primitive unit cell and therefore six phonon modes are produced in the dispersion relations. There are three dimensions $(\mathrm{d}=3)$, and thus three acoustic modes and $\mathrm{d}$ (n-1) equals three optical modes. The calculated acoustic and optical modes are in excellent agreement with the experiment done by Borcherds, et al. [28] at room (300 K) temperature.

The density of states plots in Figure $2 \mathrm{~b}$ clearly indicates that in the region around $315-390 \mathrm{~cm}^{-1}$ the phonon states are densely populated. The sharp peak in phonon density of states of $\mathrm{GaP}$ is due to the flat transverse optical branch. The gap between the acoustic and optical branch is the region where frequencies are not allowed to be propagated, and this occurred because of the difference in masses of two atoms. In the case of $\mathrm{GaP}$, this phonon gap occurs at $\sim 250-315 \mathrm{~cm}^{-1}$. The accurate prediction of phonon density of states for a variable lattice constant allows us to predict the thermodynamic properties as a function of temperatures such as the coefficient of linear thermal expansion $(\alpha)$ and heat capacity at fixed volume $\left(\mathrm{C}_{v}\right)$ correctly.

Thermal properties: The DFPT combined with QHA, at relatively low temperatures, is a powerful method to predict the lattice assisted properties. The vibrational Helmholtz free energy is calculated by evaluating the phonon density of states at different volume [12], integrating the energy of phonons over the density of states and weighting it by the temperature-dependent Boltzmann factor $[29,30]$. The thermal expansion over a wide temperature range is an important design parameter for high-temperature applications especially in choosing the post growth cooling schedule in GaP-based components.

Figure 3 a shows the change in volume of $\mathrm{GaP}$ as a function of temperature in comparison with the experimental data by Deus, et al. [31]. Comparison of the results indicates that at a temperature lower than $250 \mathrm{~K}$ the calculated volume expansion is in excellent agreement with experiment. However, at $900 \mathrm{~K}$ temperature, the value is slightly overestimated by $2.2 \%$. Figure $3 \mathrm{~b}$ shows the heat capacity at constant volume $\left(\mathrm{C}_{v}\right)$ as a function of temperature, and the calculated values are in good comparison with available data by Lui $\mathrm{Li}$, et al. [6].

\section{Lattice thermal conductivity}

Thermal conductivity, in applications involving thermal management, is an important material property to

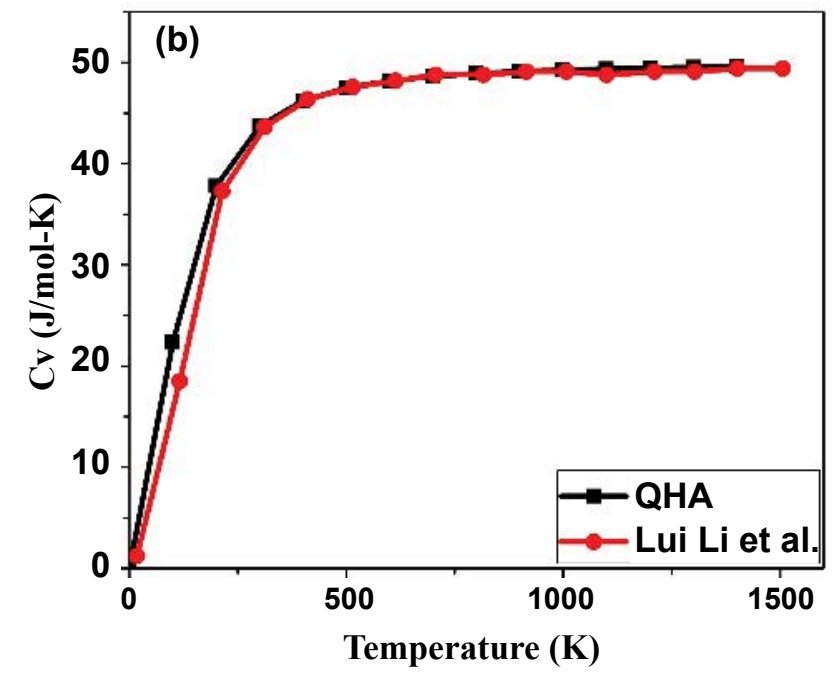

Figure 3: a) Shows the comparison of thermal expansion of GaP with experimental data from P. Deus [31]; b) Shows the comparison of $\mathrm{C}_{\mathrm{v}}$ from QHA with previous DFT calculation [6].

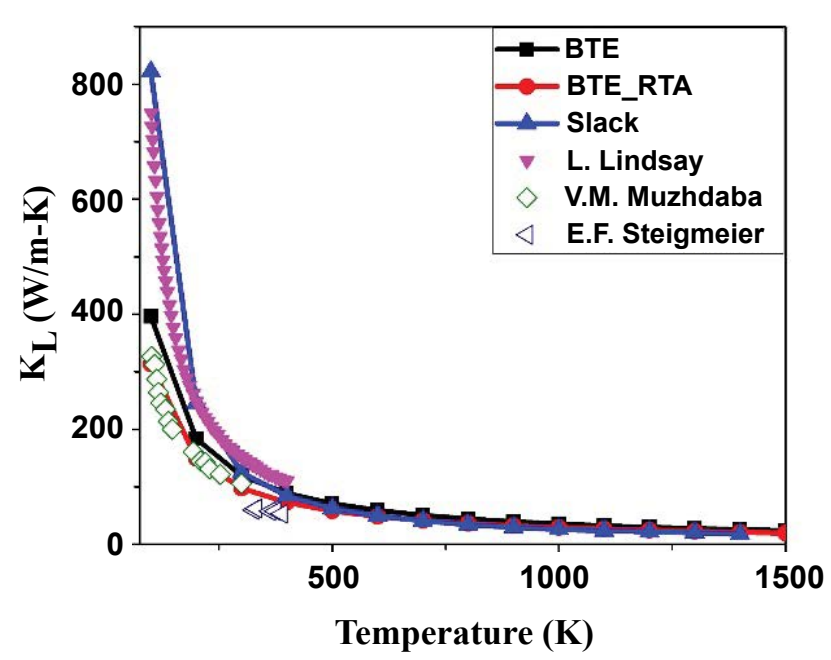

Figure 4: Comparison of calculated thermal conductivity of GaP with other simulation [8] and experimental values [4,36].

be considered. In nonmagnetic materials, the transfer of heatis governed by phonons, electrons, and radiation energy at very high temperatures. In the usually studied temperature range, the main contribution to thermal conductivity in semiconductors is made by lattice vibrations (phonons) while contributions from electrons and radiation are negligible. Therefore, in this work, we calculate only the lattice contribution to thermal conductivity $\left(\mathrm{k}_{\mathrm{L}}\right)$ of $\mathrm{GaP}$. Various models have been considered to evaluate thermal conductivity depending on the mechanisms of phonon scattering. Different methods [13] can be used to calculate the value of $k_{L}$ for materials, and such method includes Relaxation Time Approximation (RTA) [32], Callaway model [33], Slack model [13] and Boltzmann Transport Equation (BTE) [16]. In this work, we calculate the lattice thermal conductivity $\left(\mathrm{k}_{\mathrm{L}}\right)$ of $\mathrm{GaP}$ using both Slack model and Boltzmann Transport Equa- 
tion (BTE) using both RTA and iterative method solution with the inclusion up to three phonons scattering [16]. We compare our results using these methods with available experimental data.

Figure 4 shows that the $\mathrm{k}_{\mathrm{L}}$ predicted by the Slack model at lower temperatures is overestimated compared with the experimental measurement by Muzhdaba, et al. [4]. However, close to the Debye temperature ( $467 \mathrm{~K}$ ) the calculated $\mathrm{k}_{\mathrm{L}}$ shows excellent agreement with the experimental data [4]. The high value of $k_{L}$ at low temperatures is because the Slack method does not consider quasi-momentum conserving (normal process), which becomes important at low temperatures but represents well high-temperature behavior due to dominated Umklapp scattering. Although, as it is well known that $\mathrm{N}$ process alone does not lead to final conductivity. However, it is important to be included especially at lower temperature as it leads to the redistribution of the phonon momentum [34]. The Slack model predictions are better at a relatively high temperature near to the Debye temperature where the non-quasi-momentum conserving Umklapp process is dominant.

We get a good prediction for overall temperature range using BTE because it considers the boundary scattering, normal process, and the Umklapp process. Figure 4 also compares the calculation in the RTA as well as the full BTE solution. The comparison shows that the BTE exact solution is $18 \%$ higher than the RTA solution. It has been reported that any difference greater than $10 \%$ indicates that $\mathrm{N}$ scattering process is dominant [35]. Also, we calculated the percentage isotope effect for isotope composition of $\mathrm{GaP}$ to be less than $6 \%$ using the contribution to phonon scattering from isotopic disorder as implemented in Sheng BTE [16]. At low temperature, the $\mathrm{k}_{\mathrm{L}}$ predicted by BTE compares well with the experiment done by Muzhdaba, et al. [4]. Moreover, our prediction of $\mathrm{k}_{\mathrm{L}}$ from BTE are in better agreement with both experimental data from Muzhdaba, et al. [4] and Steigmeier, et al. [36] than the work done by Lindsay, et al. [8]. We have also extended the prediction of $\mathrm{k}_{\mathrm{L}}$ to relatively higher temperatures than in previous calculations. Analytically, $\mathrm{k}_{\mathrm{L}}$ is expressed well as a function of $[\mathrm{a}+\mathrm{bT}]-1$, even at higher temperatures where Umklapp scattering is dominant, with constant a and b. For GaP, we obtained $(\mathrm{R} 2=0.9488)$ the analytical expression of $\mathrm{k}_{\mathrm{L}}$ $=[4.45 \times 10-11(\mathrm{mK} / \mathrm{W})+3.07 \times 10-05(\mathrm{~m} / \mathrm{W}) \mathrm{T}(\mathrm{K})]$ -1 from the simulated values of $k_{\mathrm{L}}$ using Slack method.

\section{Conclusion}

In conclusion, we have predicted the structural, electronic, mechanical and thermal properties of the $\mathrm{GaP}$ cubic phase using new PBE sol functional. The evaluated properties in comparison with experiments are significantly more accurate than the previous DFT calculations.
We have predicted that $\mathrm{GaP}$ is a semiconductor with a slightly under predicted band gap of $1.7 \mathrm{eV}$ as expected within DFT approximation. The calculated phonon dispersion relation of $\mathrm{GaP}$ also the thermal conductivity of $\mathrm{GaP}$ cubic phase, using two complementing methods: The Slack model and BTE. For the first time, we have predicted the thermal conductivity of GaP at relatively high temperatures and our predictions are more accurate than the previously reported data and therefore they are in better agreement with experiment. The knowledge of thermal expansion, heat capacity and thermal conductivity in the broad range of temperatures would help in designing devices that can work in extreme conditions. Also, our finding of thermo-mechanical properties of $\mathrm{GaP}$ at high temperatures can be used as a complementary data in the design of devices involving thermal management.

\section{Acknowledgement}

We acknowledge access to high-performance supercomputers, Compute Canada (Westgrid and CalculQuebec), and Plato at the University of Saskatchewan. We thank Dr. Jochym Pawel for the development of QEnipy, the initial version of the software used here. We also recognize the access to Quantum ESPRESSO code and the development of QECalc by Nikolay Markovskiy and Alexander Dementsov and the development of the ASE QE interface by Luca Tornatore. The authors gratefully acknowledge the financial support of Canadian Natural Sciences and Engineering Research Council (NSERC) and Canada Research Chair program.

\section{References}

1. T Luo, J Garg, J Shiomi, K Esfarjani, G Chen (2013) Gallium arsenide thermal conductivity and optical phonon $\mathrm{Re}-$ laxation times from first-principles calculations. EPL 101: 1-6.

2. MR Lorenz, GD Pettit, RC Taylor (1968) Band gap of gallium phosphide from 0 to $900 \mathrm{~K}$ and light emission from diodes at high temperatures. Phys Rev 171: 876-881.

3. S Wang (2009) Studies on thermodynamic properties of III-V compounds by first-principles response-function calculation. Basic Solid-State Physics 246: 1618-1627.

4. SS Muzhdaba, VM Nashel'skii, AY Tamarin, PV Shalyt (1969) Thermal conductivity and thermo-emf of A/Sb and GaP at low temperatures. Sov Phys Solid State 10: 22652266.

5. N Shulumba, Z Raza, O Hellman, E Janzén, IA Abrikosov, et al. (2016) Impact of anharmonic effects on the phase stability, thermal transport, and electronic properties of AIN. Physical Review B 94: 1-8.

6. L Liu, JJ Wei, XY An, XM Wang, HN Liu, et al. (2011) Investigations of phase transition, elastic and thermodynamic properties of GaP by using the density functional theory. Chinese Physics B 20.

7. R Ahmed, Fazal e Aleem, SJ Hashemifar, H Akbarzadeh 
(2008) First-principles study of the structural and electronic properties of III-phosphides. Physica B: Condensed Matter 403: 1876-1881.

8. L Lindsay, DA Broido, TL Reinecke (2013) Ab initio thermal transport in compound semiconductors. Phys Rev B 87: 1-15.

9. P Giannozzi, S Baroni, N Bonini, M Calandra, R Car, et al. (2009) Quantum Espresso: a modular and open-source software project for quantum simulations of materials. J Phys Condens Matter 21: 39.

10. L Malakkal, B Szpunar, JC Zuniga, RK Siripurapu, JA Szpunar (2015) An Interface to Quantum Espresso. 3rd World Congress on Integrated Computational Materials Engineering (ICME 2015), John Wiley \& Sons, 155-162.

11. FD Murnaghan (1944) The compressibility of media under extreme pressure. Proc Natl Acad Sci U S A 30: 244-247.

12. Ma Blanco, E Francisco, V Luaña (2004) GIBBS: Isothermal-isobaric thermodynamics of solids from energy curves using a quasi-harmonic Debye model. Comput Phys Commun 158: 57-72.

13. DT Morelli, GA Slack (2006) High lattice thermal conductivity solids. High Therm Conduct Mater 37-68.

14. L Malakkal, B Szpunar, RK Siripurapu, JA Szpunar (2017) Thermal conductivity of bulk and nanowire of cubic-SiC from ab initio calculations. Comput Mater Sci 128: 249-256.

15. L Malakkal, B Szpunar, JC Zuniga, RK Siripurapu, JA Szpunar (2016) First principles calculation of thermo-mechanical properties of thoria using Quantum ESPRESSO. Int J Comput Mater Sci Eng 5: 1650008.

16. W Li, J Carrete, NA Katcho, N Mingo (2014) Sheng BTE: A solver of the Boltzmann transport equation for phonons. Comput Phys Commun 185: 1747-1758.

17. J Perdew, A Ruzsinszky, G Csonka, O Vydrov, G Scuseria, et al. (2008) Restoring the Density-Gradient Expansion for Exchange in Solids and Surfaces. Phys Rev Lett 100: 136406.

18. A Garcia, ML Cohen (1993) Effect of Ga 3d states on the structural properties of GaAs and GaP. Phys Rev B 47.

19. O Madelung, U Rössler, M Schulz (2001) Gallium phosphide $(\mathrm{GaP})$ lattice parameters, thermal expansion. In: Group IV Elem IV-IV III-V Compd Part a - Lattice Prop, Springer, 41: 1-9.
20. PT Jochym, K Parlinski (2000) Ab initio lattice dynamics and elastic constants of ZrC. Eur Phys J B 15: 265-268.

21. LD Landau, EM Lifshitz, AM Kosevich, LP Pitaevskii, JB Sykes, et al. (1986) Theory of Elasticity. Pergamon Press, London, 187.

22. W Voigt (1928) Lehrb Der Kriystallphysik. 962.

23. A Reuss (1929) Berechnung der fließgrenze von mischkristallen auf grund der plastizitätsbedingung für Einkristalle. ZAMM 9: 49-58.

24. R Hill (1952) The Elastic behaviour of a crystalline aggregate. Proc Phys Soc Sect A 65: 349-354.

25. D Gehrlich, M Wolf (1980) High Pressure Science and Technology.

26. JP Perdew, A Ruzsinszky, GI Csonka, OA Vydrov, GE Scuseria, et al. (2007) Generalized gradient approximation for solids and their surfaces. Phys Rev Lett 1-5.

27. RWG Wyckoff (1971) Crystal Structures. (2 ${ }^{\text {nd }}$ edn).

28. PH Borcherds, K Kunc, GF Alfrey, RL Hall (1979) The lattice dynamics of gallium phosphide. J Phys C 12: 4699-4706.

29. HY Xiao, Y Zhang, WJ Weber (2012) Thermodynamic properties of CexTh1-xO2 solid solution from first-principles calculations. Acta Mater 61: 467-476.

30. Y Lu, Y Yang, P Zhang (2012) Thermodynamic properties and structural stability of thorium dioxide. J Phys Condens Matter 24: 225801.

31. P Deus, U Voland, HA Schneider (1983) Thermal expansion of GaP within 20 to 300 K. Phys Status Solidi 80: 2932.

32. H Gzyl (1982) Integration of the boltzmann equation in the relaxation time approximation. J Stat Phys 29: 617-622.

33. J Callaway (1959) Model for lattice thermal conductivity at low temperatures. Phys Rev 113: 1046-1051.

34. S Simons (1968) Normal process relaxation times from thermal conductivity data. J Phys C Solid State Phys 1: 11.

35. A Ward, DA Broido, DA Stewart, G Deinzer (2009) Ab initio theory of the lattice thermal conductivity in diamond. Phys Rev B Condens Matter Mater Phys 80: 1-8.

36. EF Steigmeier, I Kudman (1966) Acoustical-optical phonon scattering in $\mathrm{Ge}, \mathrm{Si}$, and III-V compounds. Phys Rev 141: 767-774. 